\title{
Effect of Chromium Interlayer Thickness on Optical Properties of Au-Ag Nanoparticle Array
}

\author{
Jing Liu, ${ }^{1,2,3}$ Haoyuan Cai, ${ }^{2}$ Lingqi Kong, ${ }^{1,3}$ and Xianfang Zhu ${ }^{1,3,4}$ \\ ${ }^{1}$ Department of Physics, Xiamen University, Xiamen, Fujian 361005, China \\ ${ }^{2}$ School of Information Engineering, Jimei University, Xiamen, Fujian 361021, China \\ ${ }^{3}$ China-Australia Joint Laboratory for Functional Nanomaterials, Xiamen University, Xiamen, Fujian 361005, China \\ ${ }^{4}$ ARC Centre of Excellence for Functional Nanomaterials, University of Queensland, St Lucia, Brisbane, QLD 4072, Australia
}

Correspondence should be addressed to Xianfang Zhu; zhux@xmu.edu.cn

Received 12 June 2014; Accepted 4 August 2014; Published 19 August 2014

Academic Editor: Fu-Ken Liu

Copyright (C) 2014 Jing Liu et al. This is an open access article distributed under the Creative Commons Attribution License, which permits unrestricted use, distribution, and reproduction in any medium, provided the original work is properly cited.

\begin{abstract}
The effect of chromium interlayer thickness on optical properties of array of hybrid Au-Ag triangular nanoparticles is systematically investigated. The optical spectrum simulated by discrete dipole approximation (DDA) numerical method shows that with increase of the chromium interlayer thickness both refractive index sensitivity (RIS) and figure of merit (FOM) of localized surface plasmon resonance from the hybrid nanostructures experience remarkable change and the intensity of the extinction efficiency decreases. The nanosphere lithography (NSL) is used to fabricate the hybrid nanostructure arrays with different chromium interlayer thicknesses. The experiment demonstrates that the spectrum as measured from the as-fabricated hybrid nanostructure arrays is essentially in agreement with the simulated results.
\end{abstract}

\section{Introduction}

Noble metal nanoparticles are well known for their ability to exhibit localized surface plasmon resonance (LSPR) which can be applied in surface-enhanced spectroscopy [1], optical filters [2], plasmonic devices [3, 4], sensors [5], and so forth. The LSPR refers to the excitation of surface plasmons by light from nanometer-sized metallic particles. The position and intensity of LSPR spectrum peaks are sensitively dependent on composition, size, shape, and interparticle spacing of the nanoparticles as well as the dielectric properties of their local environments [6]. The discrete dipole approximation (DDA) is one of the most efficient computational numerical algorithms to simulate LSPR for the nanostructures with arbitrary shapes and dimensions. This method has significant advantages of occupying less computation resource, calculating the mutual action between the light and the metal nanostructures [7-10]. On the other hand, the nanosphere lithography (NSL) is the most low-cost, high-throughput method for producing periodic, geometrically tunable nanostructure arrays [11]. The
NSL makes use of a template formed by the self-assembly of monodisperse nanospheres on flat surface acting as a deposition/etching mask. Nevertheless, even with the NSL, complicated physical and chemical processes are normally involved and thus render it difficult to achieve a designed structure. One of the major problems is the adhesive ability of the deposited metallic particles to the substrate. The cohesive force and wettability of a noble metal, for example, silver or gold, are normally very limited on a glass or silicon substrate. The noble metals are inclined to be lifted off due to the abrupt change of physical and chemical properties across the interface between the metal and the substrate. Both theory and experiments suggest that an interlayer can be deposited to promote the adhesion of metallic nanoparticles to the substrates [6, 12-18]. For example, Haes et al. [6] applied chromium buffer thin film to increased adhesion of Ag nanoparticles to the glass substrate. Xilian and Jianda [13] introduced $\mathrm{Cr}$ intermediate layer, which reduced the surface roughness and promoted the reflectivity of Ag film. In addition, Zhu et al. [14] also focused on the study of 
the influence of $\mathrm{Cr}$ adhesion layer on detection of amyloidderived diffusible ligands based on localized surface plasmon resonance. To the concern of our knowledge, so far, there is no work investigating the effect of $\mathrm{Cr}$ interlayer thickness deposition on the periodic hybrid Au-Ag triangular nanoparticle array. The hybrid nanoparticles can prevent oxidation of pure Ag nanoparticles due to Au protective layer caped on the Ag nanoparticles [19-21]. The refractive index sensitivity (RIS) and figure of merit (FOM) of the LSPR sensor [22-27] are sensitive to the thickness of the Cr interlayer, so the influence of the $\mathrm{Cr}$ interlayer thickness on the optical properties and adhesive ability of the hybrid nanostructure is important as the main topic of this paper.

With above considerations, in the present paper, the effect of $\mathrm{Cr}$ interlayer thickness deposition on the periodic hybrid Au-Ag triangular nanoparticle array is systematically investigated. DDA method is used to calculate RIS and FOM of the hybrid nanostructure arrays with different $\mathrm{Cr}$ interlayer thicknesses. The hybrid nanoparticles are fabricated by NSL with polystyrene (PS) nanospheres as a deposition mask. Thermal evaporation is applied to deposit $\mathrm{Au}-\mathrm{Ag}$ and $\mathrm{Cr}$ films. The structures of the achieved hybrid nanoparticle arrays with different $\mathrm{Cr}$ interlayer thicknesses are characterized by scanning electron microscope (SEM). The results suggest that the adhesive ability of the hexagonally arranged triangular Au-Ag nanoparticles to silicon substrate can be remarkably promoted by introducing a certain thickness of $\mathrm{Cr}$ interlayer. According to the theoretical calculations and experimental results, we obtain a suitable $\mathrm{Cr}$ interlayer thickness.

\section{DDA Method}

The DDA provides a convenient method for describing light scattering from nanoparticles of arbitrary shape. In DDA formalism, the object of interest, usually called "target," is described as a cubic array lattice of electric dipoles $(N$ point dipoles) in which the polarizability and position vector of each dipole are specified as $\alpha_{i}$ and $\mathbf{r}_{i}$, respectively. The induced dipole polarization $\mathbf{P}_{i}$ in each element is determined from [7-10]

$$
\mathbf{P}_{i}=\alpha_{i} \mathbf{E}_{\mathrm{loc}, i}\left(\mathbf{r}_{i}\right), \quad i=1,2, \ldots, N,
$$

where the local field $\mathbf{E}_{\mathrm{loc}, i}\left(\mathbf{r}_{i}\right)$ is the sum of the field radiated from all of the other $N-1$ dipoles. For a given wavelength $\lambda$, including the contribution of all of the other dipoles, the local field can be written as

$$
\mathbf{E}_{\mathrm{loc}, i}\left(\mathbf{r}_{i}\right)=\mathbf{E}_{0} \exp \left(i \mathbf{k} \cdot \mathbf{r}_{i}\right)-\sum_{\substack{j=1 \\ i \neq j}}^{N} \mathbf{A}_{i j} \mathbf{P}_{j}, \quad i=1,2, \ldots, N,
$$

where $\mathbf{k}$ and $\mathbf{E}_{0}$ are the wave vector and the amplitude of the incident radiation, respectively. The contribution to the electric field at position $i$ due to the dipole at position $j$ is contained in the second term on the right side of (2) and is normally expressed in terms of the dipole-dipole interaction matrix $\mathbf{A}$ as

$$
\begin{gathered}
\mathbf{A}_{i j} \mathbf{P}_{j}=\frac{\exp \left(i \mathbf{k} r_{i j}\right)}{r_{i j}^{3}}\left\{\mathbf{k}^{2} \mathbf{r}_{i j} \times\left(\mathbf{r}_{i j} \times \mathbf{P}_{j}\right)+\frac{1-i \mathbf{k} r_{i j}}{r_{i j}^{2}}\right. \\
\left.\times\left[r_{i j}^{2} \mathbf{P}_{j}-3 \mathbf{r}_{i j}\left(\mathbf{r}_{i j} \cdot \mathbf{P}_{j}\right)\right]\right\} \\
i=1,2, \ldots, N, j=1,2, \ldots, N, j \neq i,
\end{gathered}
$$

where $\mathbf{r}_{i j}$ and $r_{i j}$ correspond to the dipole-dipole position difference vector and magnitude that are defined as $\mathbf{r}_{i}-\mathbf{r}_{j}$ and $\left|\mathbf{r}_{i}-\mathbf{r}_{j}\right|$, respectively. Substituting (2) and (3) into (1), we can generate the system of equations

$$
\mathbf{A}^{\prime} \cdot \mathbf{P}=\mathbf{E},
$$

where the off-diagonal elements of the matrix $A_{i j}^{\prime}$ are the same as $A_{i j}$, and the diagonal elements of the matrix $A_{i j}^{\prime}$ are $\alpha^{-1}$.

For a system with total dipoles, $N, \mathbf{E}$ and $\mathbf{P}$ are $3 N$ dimensional vectors, and $\mathbf{A}^{\prime}$ is a $3 N \times 3 N$ matrix. Solving this set of $3 N$ complex linear equations, the polarizations $\mathbf{P}_{i}$ are determined, and from this we can calculate cross section of the extinction as

$$
C_{\mathrm{ext}}=\frac{4 \pi \mathbf{k}}{\left|\mathbf{E}_{0}\right|^{2}} \sum_{i=1}^{N} \operatorname{Im}\left(\mathbf{E}_{\mathrm{loc}, i}^{*} \cdot \mathbf{P}_{i}\right) .
$$

\section{Results and Discussions}

Using the DDA algorithm, we design and calculate the extinction spectra, RIS, and FOM of the hybrid Au-Ag triangular nanostructure arrays with different $\mathrm{Cr}$ interlayer thicknesses. Corresponding schematic illustration of the hybrid nanoprism is shown in Figure 1. The triangle in XY plane contains three equilateral edges, each of which is of $180 \mathrm{~nm}$. The thickness of Au and Ag is fixed on $h_{\mathrm{Au}}=5 \mathrm{~nm}$ and $h_{\mathrm{Ag}}=25 \mathrm{~nm}$ and the Cr interlayer thickness, $h_{\mathrm{Cr}}$, is varying from $4 \mathrm{~nm}$ to $20 \mathrm{~nm}$. Here $h_{\mathrm{Au}}, h_{\mathrm{Ag}}$, and $h_{\mathrm{Cr}}$ are defined in Figure 1(b).

Extinction is a term used in physics to describe the absorption and scattering of electromagnetic radiation. Any changes in the parameters of metal nanoparticles could lead to the optical drift in extinction spectrum and thus influence the optical applications in practice [28-31]. According to the calculated extinction spectra presented in Figure 2, when the $\mathrm{Cr}$ interlayer thickness is increased, the positions of peak wavelengths appear slightly blue shifted while the intensity of the extinction has obviously been decreased. The full width at half maximum (FWHM) increases rapidly by increasing thickness of $\mathrm{Cr}$ interlayer from 4 to $16 \mathrm{~nm}$ and then increases slowly with increasing $\mathrm{Cr}$ interlayer thickness to $20 \mathrm{~nm}$. It is noted that, as can be seen from the extinction spectrum in Figure 2(a), each curve has several peaks independent of the thickness of $\mathrm{Cr}$. This phenomenon not only relates to the hybrid Au and Ag material, but also relates to the optical 


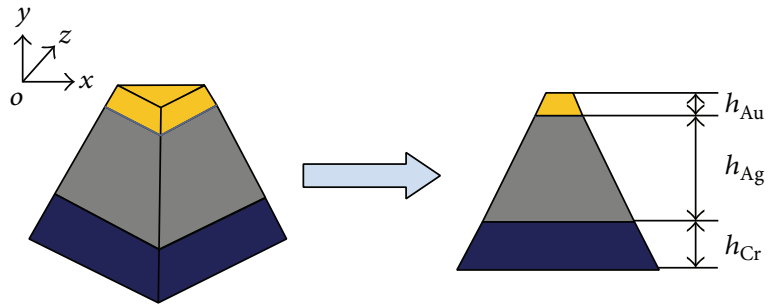

FIGURE 1: Schematic view of a single hybrid Au-Ag triangular nanoparticle.

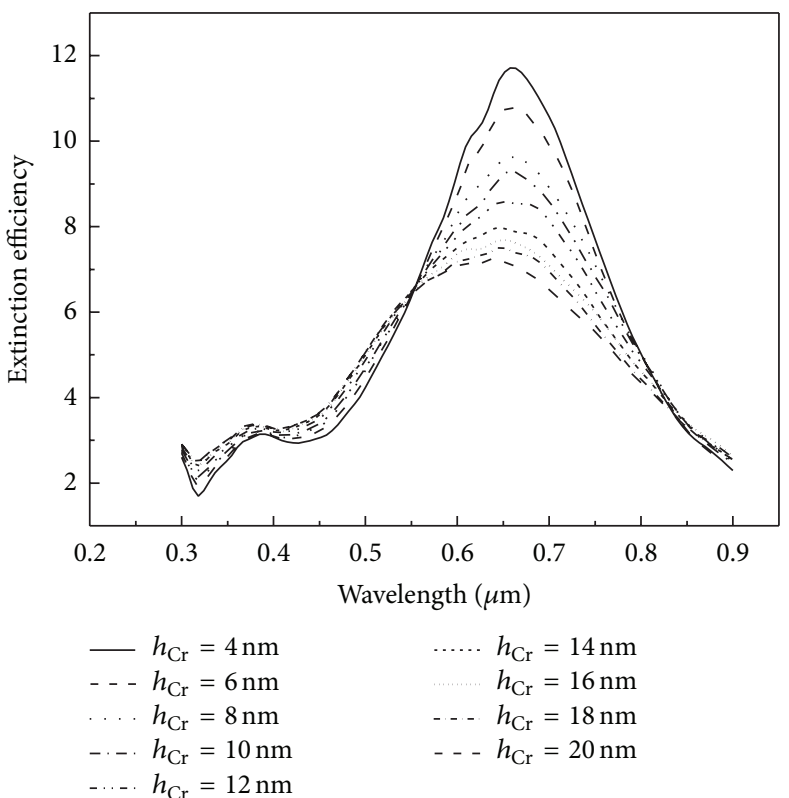

(a) Extinction spectra for different thickness of the Cr film

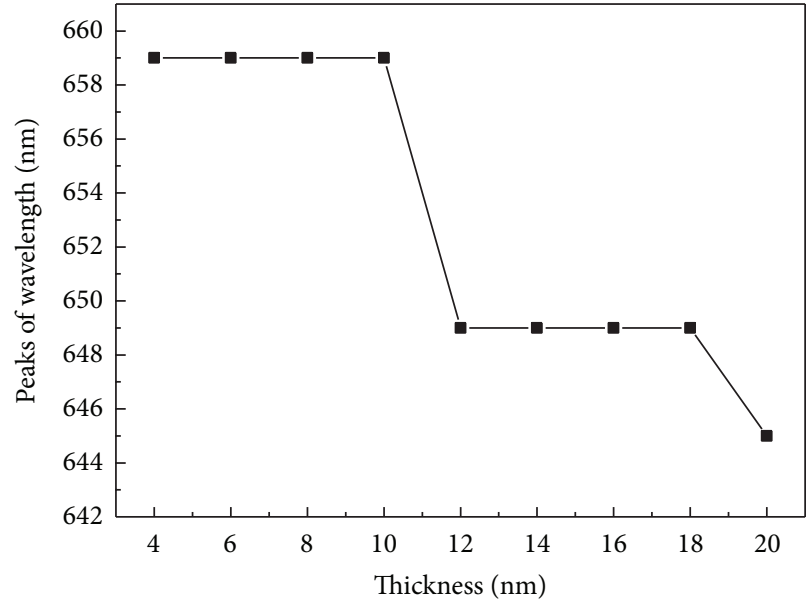

(b) Peak wavelength

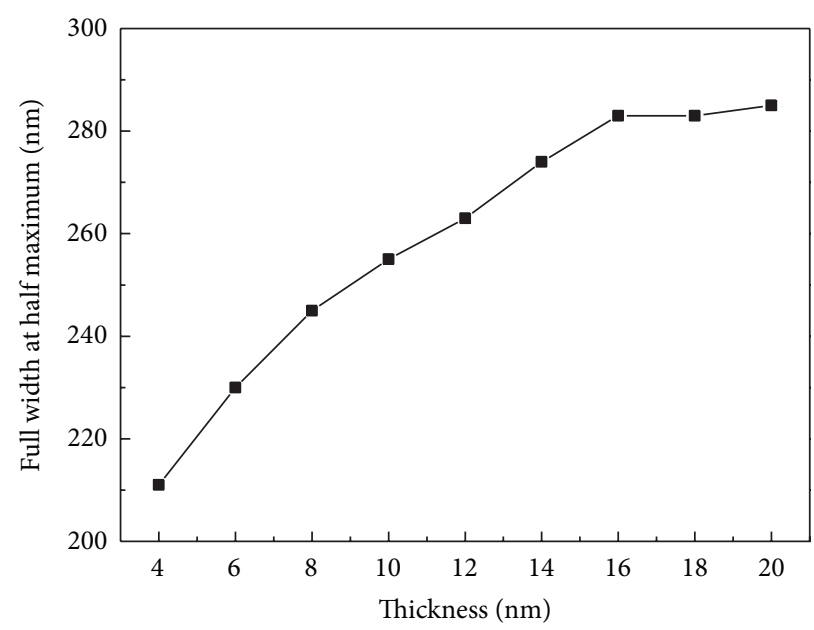

(c) Full width at half maximum as a function of the thickness of Cr film

FIgURE 2: Calculation results ofthe hybrid Au-Ag triangular nanoparticle arrays with different Cr interlayer thicknesses.

properties of triangular nanoparticle. For Ag or Au triangular nanoprism, the extinction spectrum always shows several peaks due to the in-plane and out-of-plane polarization [32].

In order to investigate the effect of the $\mathrm{Cr}$ interlayer thickness on the sensitivity of the hybrid nanostructure array, we calculated the extinction spectra of the effective refractive index of the medium surrounding the nanostructure array. The RIS is defined as $m=\Delta \lambda / \Delta n$ [33], where $\Delta \lambda$ and $\Delta n$ denote the peak of the wavelength change and the refractive index change, respectively. For the hybrid nanostructure 


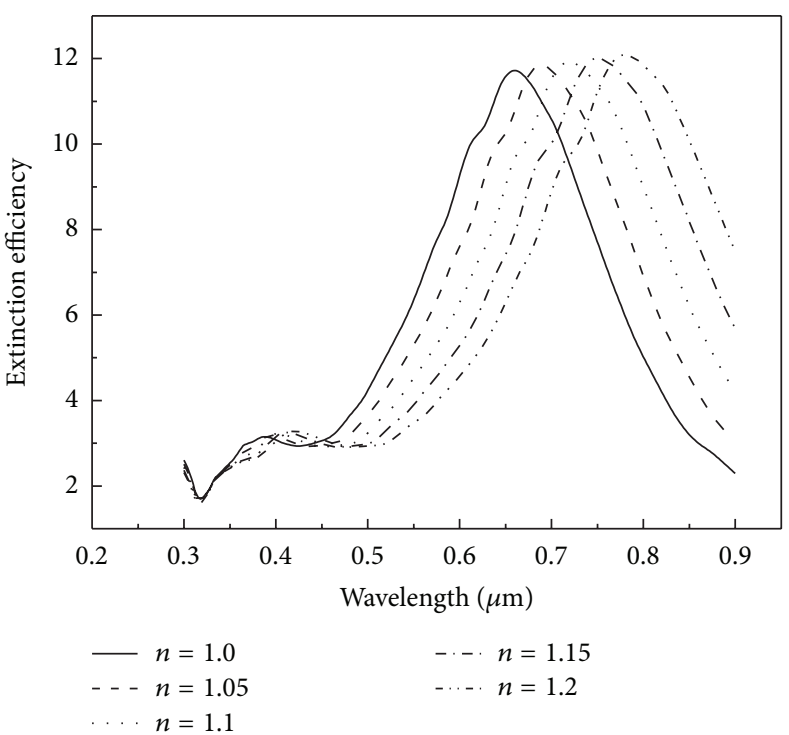

(a) Extinction spectra in different media for $h_{\mathrm{Cr}}=4 \mathrm{~nm}$

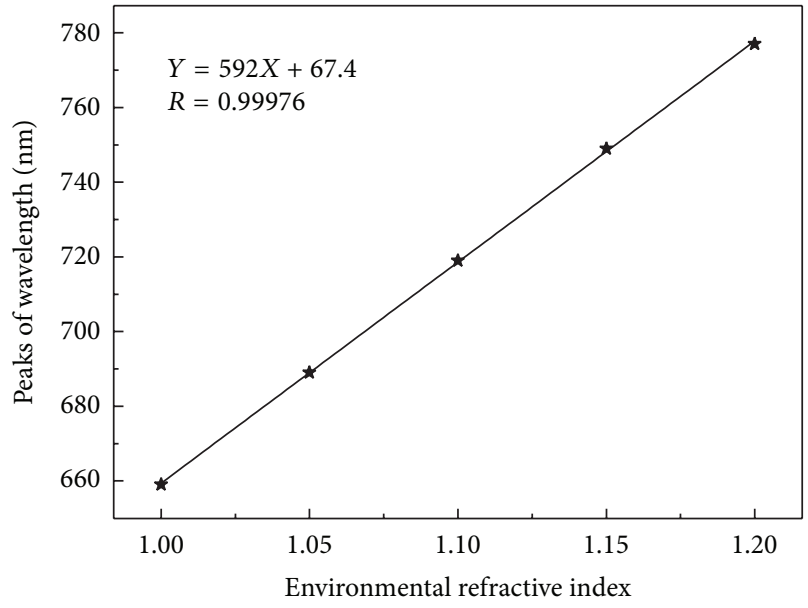

(b) Refractive index sensitivity curve for $h_{\mathrm{Cr}}=4 \mathrm{~nm}$

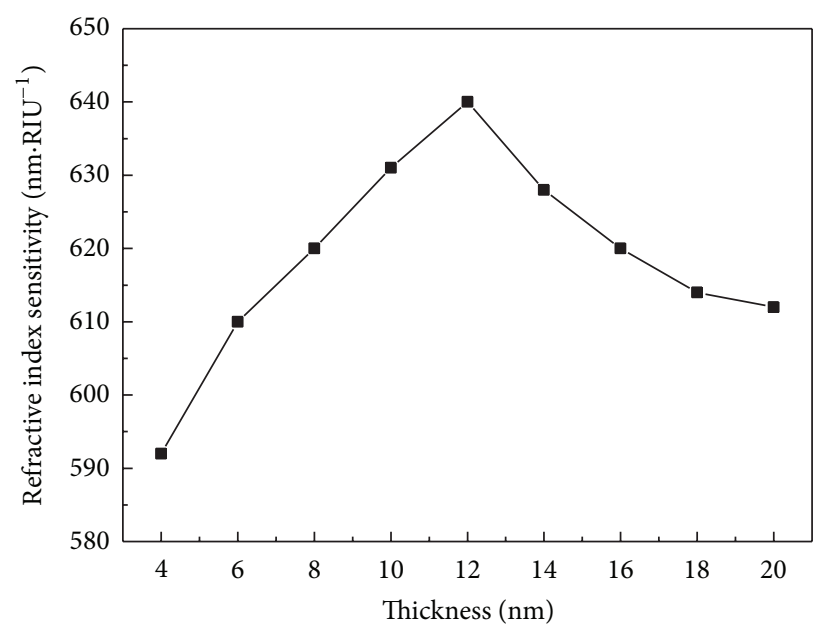

(c) Refractive index sensitivity distributions for different $\mathrm{Cr}$ interlayer thicknesses

FIGURE 3: Sensing performance of the hybrid Au-Ag triangular nanoparticle arrays with different Cr interlayer thicknesses.

array with $4 \mathrm{~nm}$ Cr interlayer thickness, the peak wavelength has a red shift when the refractive index $n$ increases as shown in Figure 3(a). For example, when the refractive index increases from 1.0 to 1.05 , the peak wavelength shifts from $659 \mathrm{~nm}$ to $689 \mathrm{~nm}$, exhibiting a refractive index sensitivity of $592 \mathrm{~nm} / \mathrm{RIU}$ (refractive index unit), as indicated in Figure 3(b). Figure 3(c) shows the RIS of the hybrid nanostructure arrays with different $\mathrm{Cr}$ interlayer thicknesses. The result shows that the RIS increases with the thickness of $\mathrm{Cr}$ interlayer from 4 to $12 \mathrm{~nm}$ and then decreases with further increase of $\mathrm{Cr}$ interlayer thickness to $20 \mathrm{~nm}$. When the Cr interlayer thickness is increased from 10 to $14 \mathrm{~nm}$, the structure is very sensitive for the refractive index from 1.0 to 1.2 , and the RIS is higher than the other thickness.

The FOM for a metal nanostructure is defined as $p=S / W$ [34], where $S$ and $W$ denote the RIS and FWHM, respectively.
According to Figures 2(c) and 3(c), we calculated the FOM and the calculation result is shown in Figure 4. It can be seen from Figure 4 that the thickness of the $\mathrm{Cr}$ interlayer causes the change of the FOM of the nanostructure array. The FOM generally decreases with the increasing thickness of Cr thickness; moreover, the FOM at 4-8 nm and 12-16 nm shows more abrupt change than those at $8-12 \mathrm{~nm}$ and $16-$ $20 \mathrm{~nm}$. From the calculated results, we can see that the FOM of the hybrid nanostructure array keeps at a more stable and relatively high value (the value less descended), when the thickness of $\mathrm{Cr}$ interlayer increases from 8 to $12 \mathrm{~nm}$.

\section{Fabrication of Nanostructures}

The PS nanospheres with a mean diameter of $360 \mathrm{~nm}$ and a concentration of $10 \mathrm{wt} \%$ in solution are purchased from 


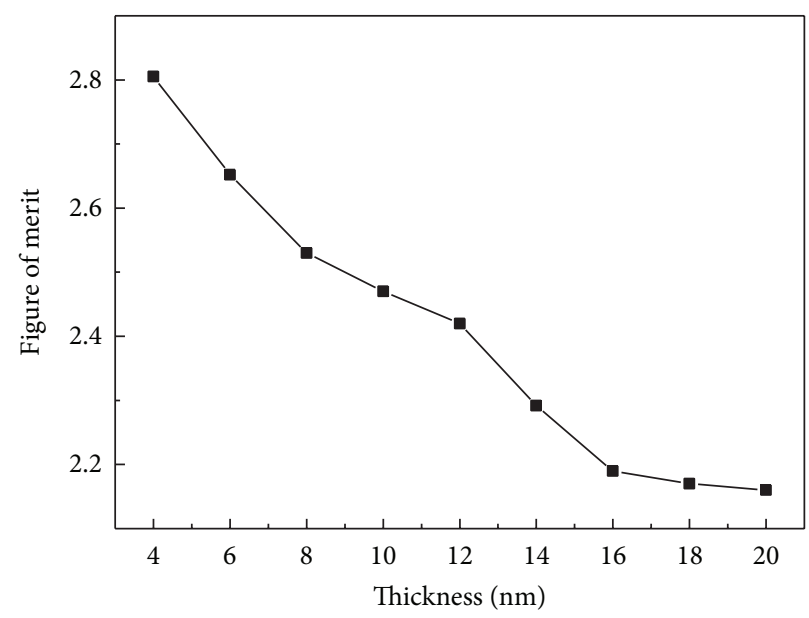

Figure 4: Figure of merit of the hybrid Au-Ag triangular nanoparticle arrays with different Cr interlayer thicknesses.

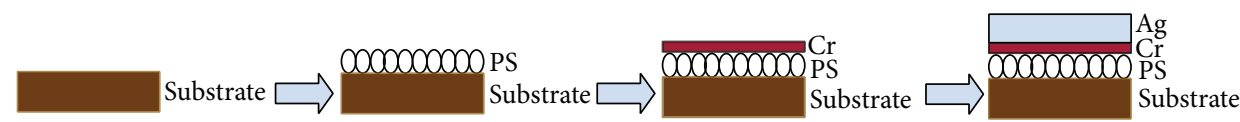

(1)

(2)

(3)

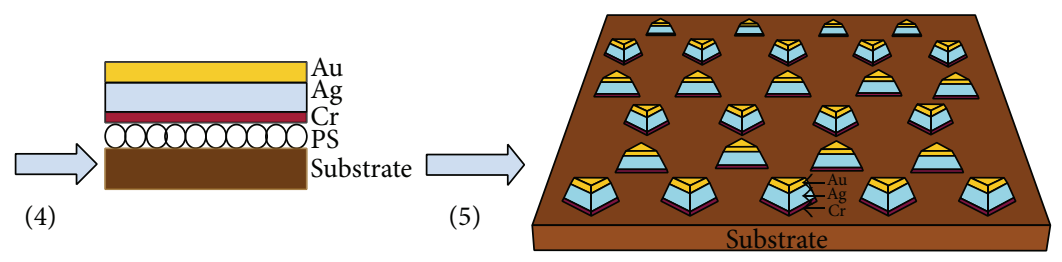

FIGURE 5: Schematic illustration of hybrid Au-Ag nanoparticle array fabrication with the following five steps: (1) drop-coating of monolayered PS nanospheres on substrate; (2) deposition of a thin buffer layer of Cr film over the as-coated monolayered PS nanospheres; (3) a further deposition of a layer of Ag film following the Cr deposition; (4) final deposition of a caped layer of Au thin film on top of the Ag film; (5) a 2D hybrid Au-Ag nanoparticle array formation on substrate after left-off of the monolayered PS nanospheres.

Suzhou Nano-Micro Bio-Tech Co. Ltd. First of all, closepacked nanosphere is a prerequisite. The regular monolayer as a deposition mask is principal to achieve large-area hexagonal structure. To begin with, the PSNS solution is diluted to be $3 \mathrm{wt} \%$ with deionized water. The silicon substrates ( $n$-type, (100) orientation) are firstly ultrasonic cleaned, in toluene, acetone, and ethanol for $10 \mathrm{~min}$, respectively, and then in piranha solution $\left(\mathrm{H}_{2} \mathrm{SO}_{4}: \mathrm{H}_{2} \mathrm{O}_{2}=3: 1\right)$ for 2 hours to remove organic residues. To achieve a hydrophilic surface, the silicon substrates are ultrasonically bathed in $\mathrm{NH}_{4} \mathrm{OH}, \mathrm{H}_{2} \mathrm{O}_{2}$, and $\mathrm{H}_{2} \mathrm{O}$ solution with the ratio of $1: 1: 5$ for 2 hours. Every sonication followed rinsing with large amount of deionized water. The cleaned substrates are stored in deionized water until used.

Following drop-coating of the PS nanospheres on the substrate, $\mathrm{Cr}, \mathrm{Ag}$, and Au layers are deposited on the PS mask sequentially, as shown in Figure 5. When the $\mathrm{Cr}$ interlayer is deposited on the PS mask, the tips of the acquired triangle nanoparticles are much sharper, and the density array is much larger than the other cases. It is believed that the structure of the 2D hybrid $\mathrm{Au}-\mathrm{Ag}$ nanoparticle array with $\mathrm{Cr}$ interlayer is better than that without interlayer. The hybrid Au-Ag particles can protect oxidation and sulfuration of the pure $\mathrm{Ag}$ particles from ambient environment.

The deposition of three metallic layers $(3 \mathrm{~N} \mathrm{Au}, 3 \mathrm{~N} \mathrm{Ag}$, and $3 \mathrm{~N} \mathrm{Cr}$ ) is performed in a home-built thermal evaporator at a pressure of $5.0 \times 10^{-4} \mathrm{~Pa}$. The substrates are rotated at a speed of $16.5 \mathrm{rpm}$ all through the deposition. To achieve homogeneous deposition, the power for heating-up of the source materials is carefully increased. The deposition rate is $\sim 2.5 \mathrm{~nm} / \mathrm{s}$ for Au and Ag layers and $\sim 4.0 \mathrm{~nm} / \mathrm{s}$ for $\mathrm{Cr}$ film. The thickness had been monitored using a Dektak 3 Series surface profiler to achieve an identical depth for a low reflectance. It is controlled to be $5 \mathrm{~nm}$ for $\mathrm{Au}$ film, $25 \mathrm{~nm}$ for $\mathrm{Ag}$ film, 3, 4, 8, 12,20 , and $64 \mathrm{~nm}$ for Cr film, respectively. After deposition of Au film, the PS spheres are lifted off by immersing in absolute ethanol for about $5 \mathrm{~s}$. The PS spheres are also removed by sonication (B3500S-MT, Branson, $140 \mathrm{~W}, 42 \mathrm{kHz}$ ) in absolute ethanol to examine the adhesive ability of the hybrid AuAg nanoparticles on silicon substrates. Nanostructures of the achieved PS mask and the hybrid Au-Ag nanoparticle arrays are characterized by LEO-1530 SEM. Ultraviolet visible (UVvis) spectra are obtained on a Varian Cary 5000 UV-Vis-NIR spectrophotometer. 


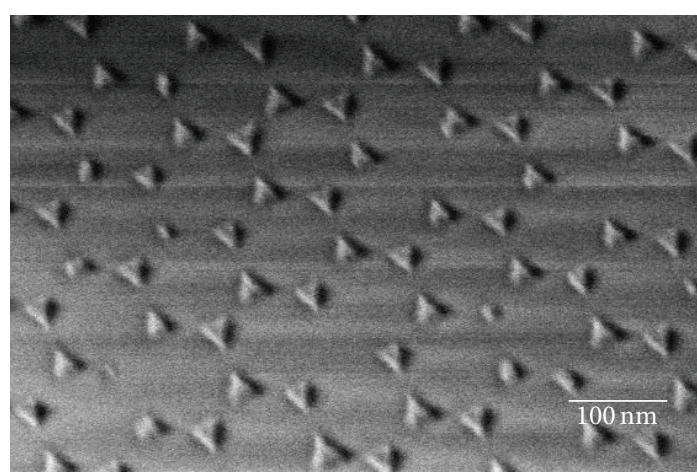

(a)

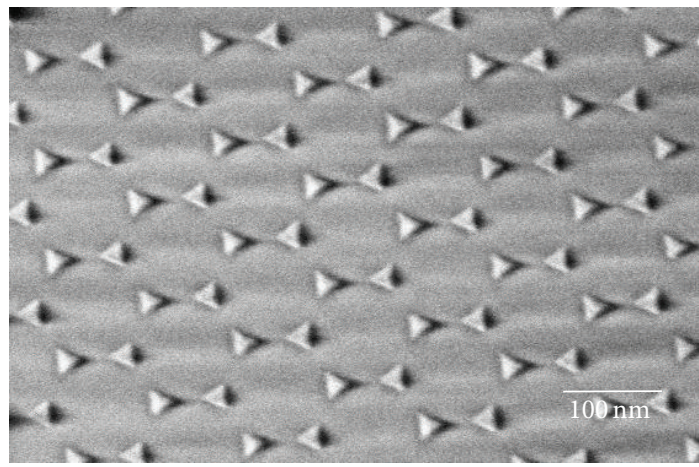

(c)

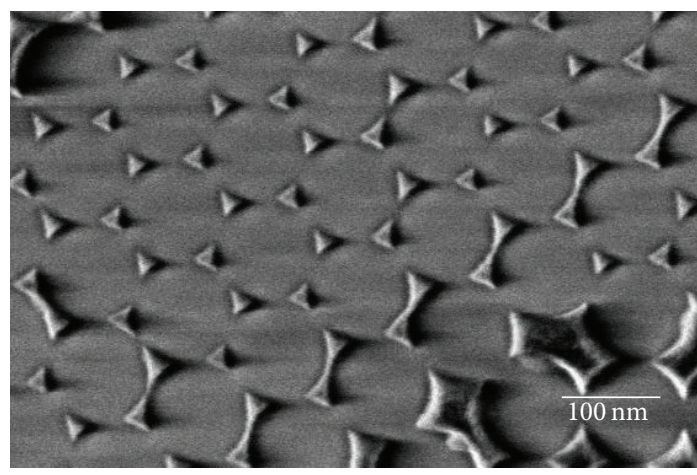

(e)

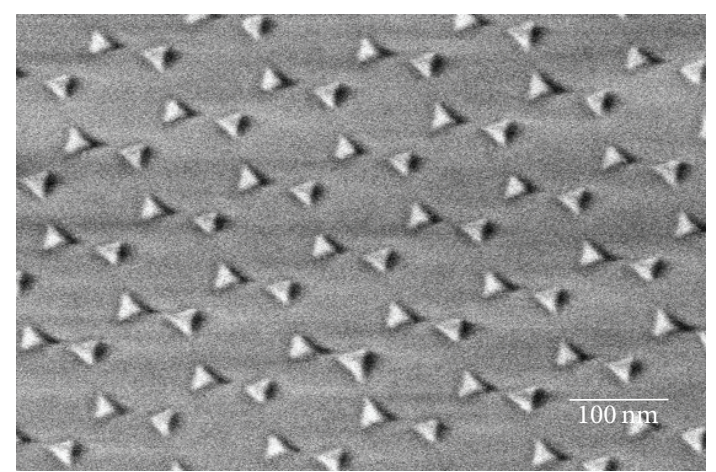

(b)

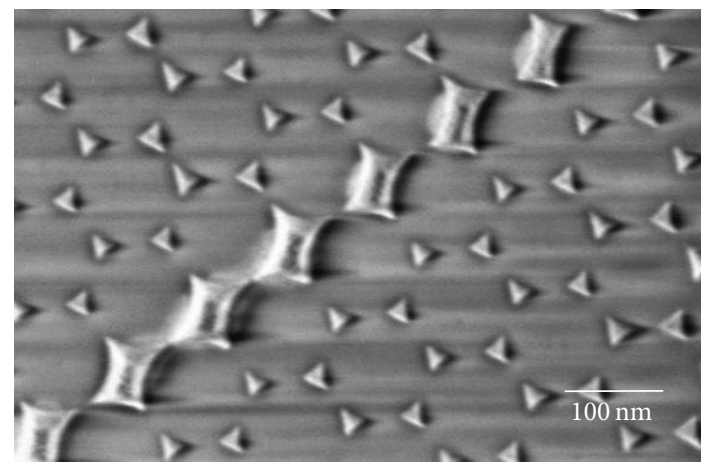

(d)

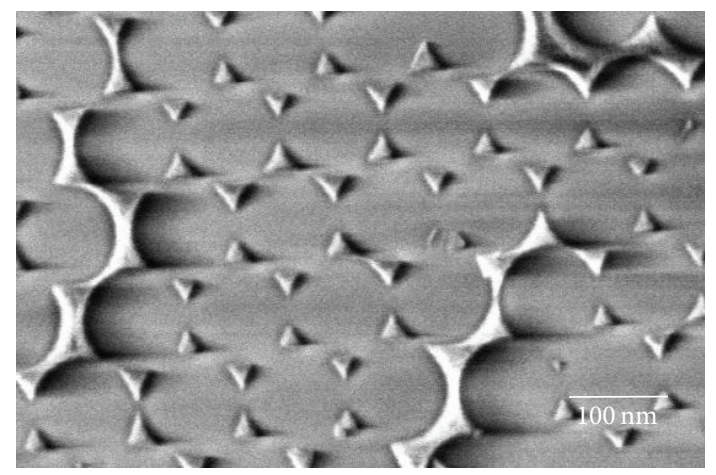

(f)

FIgURE 6: The SEM images of the Cr triangular nanoparticle arrays with different thicknesses of Cr film deposited: (a) $3 \mathrm{~nm}$, (b) $4 \mathrm{~nm}$, (c) $8 \mathrm{~nm},(\mathrm{~d}) 12 \mathrm{~nm},(\mathrm{e}) 20 \mathrm{~nm}$, and (f) $64 \mathrm{~nm}$.

\section{Results and Discussion}

Figure 6 shows the SEM images of the Cr triangular nanoparticle arrays in different thicknesses. Figure 7 shows the SEM images of the hybrid $\mathrm{Au}-\mathrm{Ag}$ triangular nanoparticles arrays with the different thicknesses of the Cr films.

As showed in Figure 7(a), the hybrid nanoparticles exhibit a hexagonally arranged disc structure rather than triangular structure and many tiny cracks appear in the structure. When the thickness of deposited Cr film is $3 \mathrm{~nm}$, the cohesive force between the PS nanospheres and the silicon substrate seems not to be strong enough, as shown in Figure 6(a). However, as the deposited Cr film thickness increases, the cohesive force between the PS nanospheres and the silicon substrate seems enhanced; thus the angular structure mounts and tends to be regular or well-defined, as shown in Figure 6(b). After the Ag and $\mathrm{Au}$ layers are deposited, the nanoparticles still exhibit similar morphology of the hybrid Au-Ag triangular nanoparticles arrays, as shown in Figure 7(b). As showed in Figure 6(c), when the Cr interlayer thickness is $8 \mathrm{~nm}$, the angular structure turns into regular shape, and its tip becomes sharp. The $\mathrm{Cr}$ interlayer of this thickness can be used to improve the structure of the hybrid Au-Ag triangular nanoparticles arrays. After the Ag and Au layers are deposited, the nanoparticles still exhibit similar morphology of the hybrid Au-Ag triangular nanoparticles arrays, as shown in Figure 7(c). As can be seen from Figures 6(d) to 6(f), with the further increase in the thickness of the predeposited $\mathrm{Cr}$ 


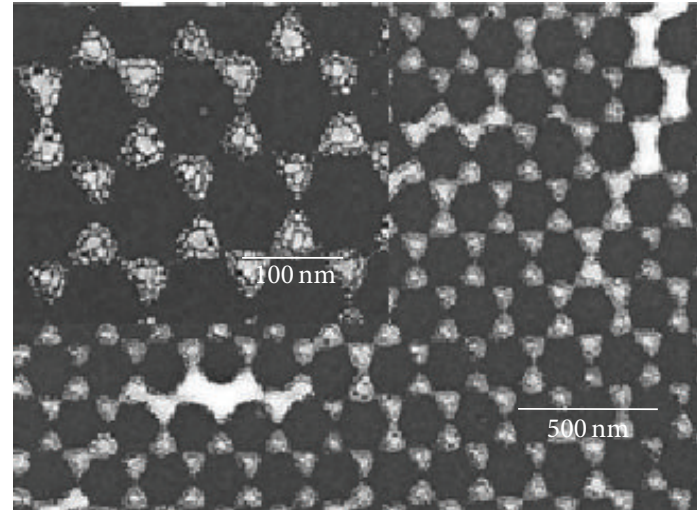

(a)

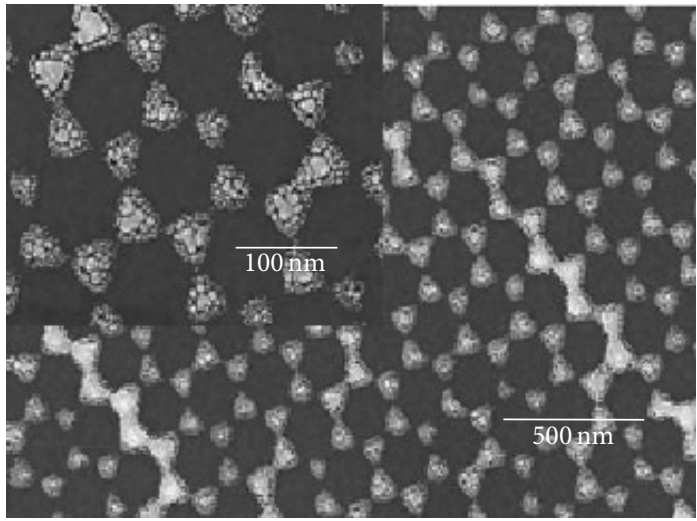

(c)

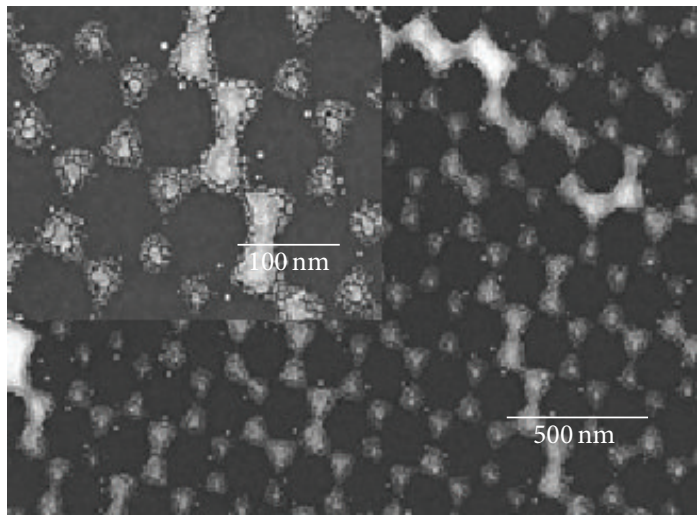

(e)

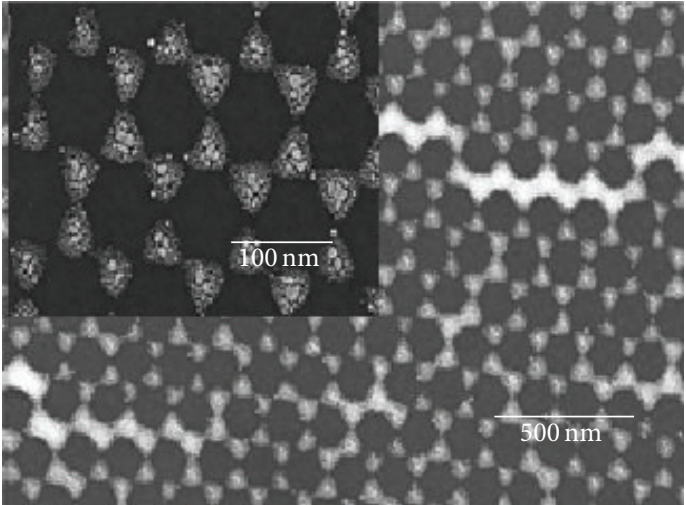

(b)

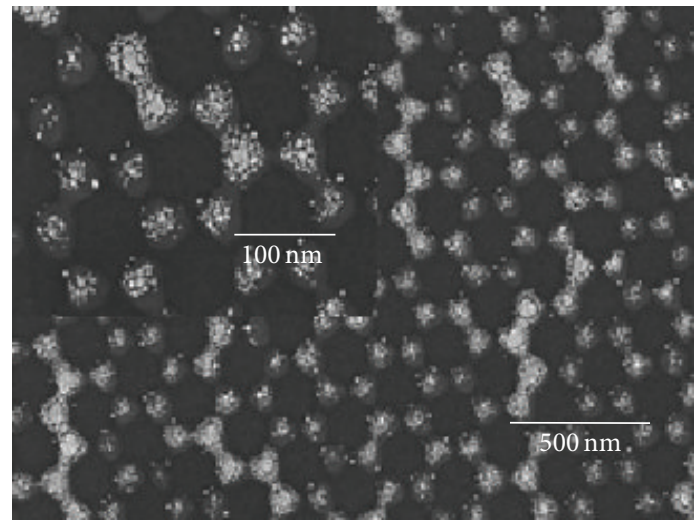

(d)

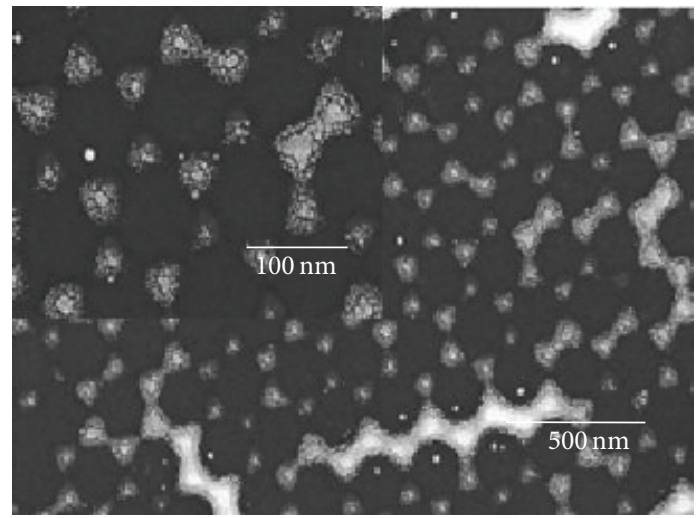

(f)

Figure 7: The SEM images of the hybrid Au-Ag triangular nanoparticle arrays; the Cr interlayer thickness is 3, 4, 8, 12, 20, and 64 nm, respectively.

film, the Ag and Au deposition in the gap interspaces among the spheres seem to become increasingly blocked with the predeposited $\mathrm{Cr}$ nanopaticles, which makes the $\mathrm{Au}$ and $\mathrm{Ag}$ particles difficult to arrive at the silicon substrate. In doing so, after the PS spheres are removed by sonication in absolute ethanol, the nanoparticles still exhibit a triangular but less uniform structure than the previous cases, as in Figures from 7 (d) to $7(\mathrm{f})$. When thickness of $\mathrm{Cr}$ is $3 \mathrm{~nm}$ and $8 \mathrm{~nm}$, the hybrid nanoparticles both form a large-scale monolayer array with the hexagonally close-packed structure. Considering the theoretical calculations and experimental results, $8 \mathrm{~nm} \mathrm{Cr}$ interlayer thickness is selected in the further experiment.

In the experiments, we detected the extinction efficiency of the representative hybrid $\mathrm{Au}-\mathrm{Ag}$ triangular nanoparticle array with $8 \mathrm{~nm}$ thickness of the $\mathrm{Cr}$ interlayer. The experimental and the calculated results are shown in Figure 8. It can be seen apparently that when the wavelength is $621 \mathrm{~nm}$, the extinction efficiency reaches a maximum value. 


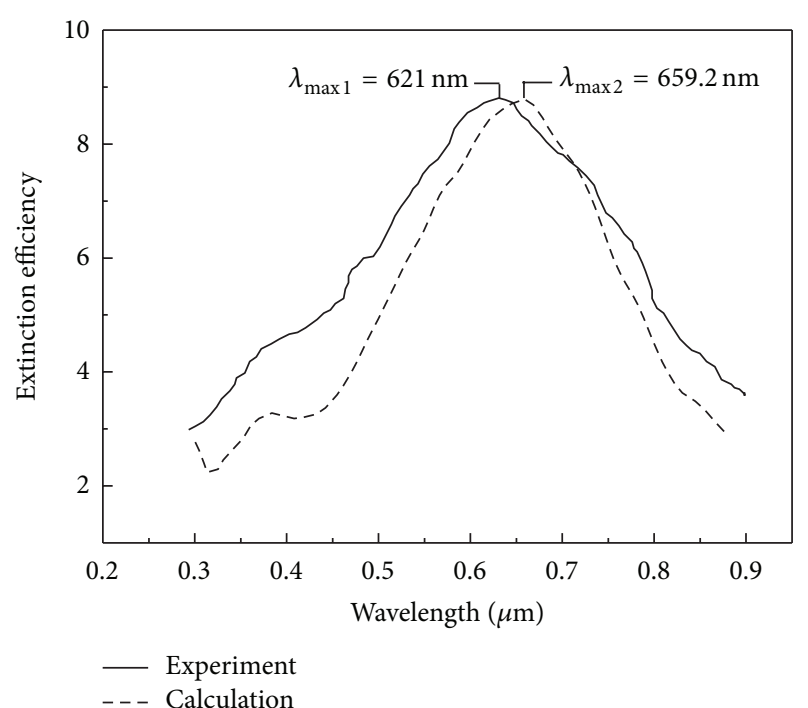

FIGURE 8: Extinction spectra of experiment and calculation.

When DDA calculation is taken to calculate the extinction efficiency, the maximum wavelength of extinction efficiency is $659.2 \mathrm{~nm}$, as shown in Figure 8. The calculated result of the plasmon wavelength with the designed model agrees with recent experimental result. The only major difference with the experiment is that the experimental peak is shifted by $38 \mathrm{~nm}$ to the blue compared to the calculated result. This is likely caused by substrate effect (see [35]). The other reason is that fabrication error causes uniformity issue for both size and shape of the particles. In addition, in DDA calculated model, the edge of the triangular nanoparticles is straight while in experiment it is curving. Most of the results presented here are analysis and characterization of the $\mathrm{Cr}$ interlayer thickness variation. The results for the $2 \mathrm{D}$ hexagonally arranged hybrid Au-Ag triangular nanoparticles arrays show that the perfect $\mathrm{Cr}$ interlayer thickness is around $8 \mathrm{~nm}$ for our experimental fabrication. The experimental results are generally in agreement with the calculated results.

\section{Summary}

The effect of the $\mathrm{Cr}$ interlayer thickness deposition on the periodic hybrid Au-Ag triangular nanostructure is systematically investigated. DDA numerical method is used to study the RIS and FOM of the hybrid nanostructures. The calculation results show that the $\mathrm{Cr}$ interlayer thickness ranging from 8 to $12 \mathrm{~nm}$ is the better geometrical parameters of the hybrid nanostructures. The NSL is used to fabricate the hybrid nanostructure arrays with different $\mathrm{Cr}$ interlayer thicknesses. The experimental results demonstrate that when the thickness is fixed on $3 \mathrm{~nm}$ and $8 \mathrm{~nm}$, the nanoparticles exhibit a triangular structure but that is more uniform than the other cases. From the above results, about $8 \mathrm{~nm}$ of $\mathrm{Cr}$ interlayer thickness is the suitable structure parameter which can be used to fabricate hybrid $\mathrm{Au}-\mathrm{Ag}$ triangular nanoparticles array with sharp tip and regular angular structure.

\section{Conflict of Interests}

The authors declare that there is no conflict of interests regarding the publication of this paper.

\section{Acknowledgments}

This work was supported by National Key Basic Science Research Program (973 Project) under Grant no. 2007CB936603, Science and Technology Cooperation Plan Key Project from Fujian Province Science and Technology Department (2014-2017), the China-MOST International Sci and Tech Cooperation and Exchange Project under Grant no. 2008DFA51230, S\&T Plan Projects of Xiamen City under Grant no. $3502 Z 20143020$ and no. 2012S0484, S\&T Plan Projects of Fujian Provincial Education Department under Grant no. JA11157/12200, and Scientific Research Foundation of Jimei University (PanJinlong) under Grant no. C512038.

\section{References}

[1] C. L. Haynes, A. D. McFarland, L. L. Zhao et al., "Nanoparticle optics: the importance of radiative dipole coupling in twodimensional nanoparticle arrays," Journal of Physical Chemistry B, vol. 107, no. 30, pp. 7337-7342, 2003.

[2] C. L. Haynes and R. P. van Duyne, "Dichroic optical properties of extended nanostructures fabricated using angle-resolved nanosphere lithography," Nano Letters, vol. 3, no. 7, pp. 939-943, 2003.

[3] P. C. Andersen and K. L. Rowlen, "Brilliant optical properties of nanometric noble metal spheres, rods, and aperture arrays," Applied Spectroscopy, vol. 56, no. 5, pp. 124A-135A, 2002.

[4] Y. Fu, W. Zhou, and L. E. N. Lim, "Near-field behavior of zoneplate-like plasmonic nanostructures," Journal of the Optical Society of America A: Optics, Image Science, and Vision, vol. 25, no. 1, pp. 238-249, 2008.

[5] A. J. Haes, S. L. Zou, G. C. Schatz, and R. P. van Duyne, "A nanoscale optical biosensor: the long range distance dependence of the localized surface plasmon resonance of noble metal nanoparticles," Journal of Physical Chemistry B, vol. 108, no. 1, pp. 109-116, 2004.

[6] A. J. Haes, W. P. Hall, L. Chang, W. L. Klein, and R. P. van Duyne, "A localized surface plasmon resonance biosensor: first steps toward an assay for Alzheimer's disease," Nano Letters, vol. 4, no. 6, pp. 1029-1034, 2004.

[7] R. Jin, Y. Cao, C. A. Mirkin, K. L. Kelly, G. C. Schatz, and J. G. Zheng, "Photoinduced conversion of silver nanospheres to nanoprisms," Science, vol. 294, no. 5548, pp. 1901-1903, 2001.

[8] B. T. Draine and P. J. Flatau, "Discrete-dipole approximation for scattering calculations," Journal of the Optical Society of America A: Optics and Image Science, and Vision, vol. 11, no. 4, pp. 14911499, 1994.

[9] N. Félidj, J. Aubard, and G. Lévi, “Discrete dipole approximation for ultraviolet-visible extinction spectra simulation of silver and gold colloids," The Journal of Chemical Physics, vol. 111, no. 3, pp. 1195-1208, 1999.

[10] G. C. Schatz, "Electrodynamics of nonspherical noble metal nanoparticles and nanoparticle aggregates," Journal of Molecular Structure, vol. 573, no. 1-3, pp. 73-80, 2001.

[11] K. H. Lee, Q. L. Chen, C. H. Yip, Q. Yan, and C. C. Wong, "Fabrication of periodic square arrays by angle-resolved nanosphere 
lithography," Microelectronic Engineering, vol. 87, no. 10, pp. 1941-1944, 2010.

[12] Y. B. Zheng, B. K. Juluri, X. Mao, T. R. Walker, and T. J. Huang, "Systematic investigation of localized surface plasmon resonance of long-range ordered Au nanodisk arrays," Journal of Applied Physics, vol. 103, no. 1, Article ID 014308, 2008.

[13] S. Xilian and S. Jianda, "Influence of Cr interlayer on the structure and optical properties of Ag films on glass substrate by magnetron sputtering," Applied Surface Science, vol. 253, no. 4, pp. 2093-2095, 2006.

[14] S. Zhu, C. Du, Y. Fu, Q. Deng, and L. Shi, "Influence of Cr adhesion layer on detection of amyloid-derived diffusible ligands based on localized surface plasmon resonance," Plasmonics, vol. 4, no. 2, pp. 135-140, 2009.

[15] G. H. Jeong, J. K. Park, K. K. Lee et al., "Fabrication of low-cost mold and nanoimprint lithography using polystyrene nanosphere," Microelectronic Engineering, vol. 87, no. 1, pp. 51$55,2010$.

[16] A. Sinitskii, S. Neumeier, J. Nelles, M. Fischler, and U. Simon, "Ordered arrays of silicon pillars with controlled height and aspect ratio," Nanotechnology, vol. 18, Article ID 305307, 2007.

[17] M. C. Gwinner, E. Koroknay, F. Liwei et al., "Periodic large-area metallic split-ring resonator metamaterial fabrication based on shadow nanosphere lithography," Small, vol. 5, no. 3, pp. 400406, 2009.

[18] Y. Imura, M. Kato, T. Kondo, and T. Kawai, "Strings of metal half-shells fabricated using colloidal particle monolayer as a template," Langmuir, vol. 26, no. 13, pp. 11314-11318, 2010.

[19] J. Liu, H. Yang, X. Luo, W. Ma, and Y. Liu, "Investigation of localized surface plasmons resonance properties of metal composition nanoparticles," Acta Optica Sinica, vol. 30, no. 4, pp. 1092-1095, 2010.

[20] Y. Fu and W. Zhou, "Hybrid Au-Ag subwavelength metallic structures with variant periods for superfocusing," Journal of Nanophotonics, vol. 3, no. 1, 2009.

[21] K. Baba, T. Okuno, and M. Miyagi, "Silver-gold compound metal island films prepared by using a two-step evaporation method," Applied Physics Letters, vol. 62, no. 5, pp. 437-439, 1993.

[22] G. K. Joshi, P. J. McClory, S. Dolai, and R. Sardar, "Improved localized surface plasmon resonance biosensing sensitivity based on chemically-synthesized gold nanoprisms as plasmonic transducers," Journal of Materials Chemistry, vol. 22, no. 3, pp. 923-931, 2012.

[23] J. S. Sekhon, H. K. Malik, and S. S. Verma, "DDA simulations of noble metal and alloy nanocubes for tunable optical properties in biological imaging and sensing," RSC Advances, vol. 3, no. 35, pp. 15427-15434, 2013.

[24] B. Spackova and J. Homola, "Sensing properties of lattice resonances of 2D metalnanoparticle arrays: an analytical model," Optics Express, vol. 21, no. 22, pp. 27490-27502, 2013.

[25] G. H. Chan, J. Zhao, G. C. Schatz, and R. P. V. Duyne, "Localized surface plasmon resonance spectroscopy of triangular aluminum nanoparticles," Journal of Physical Chemistry C, vol. 112, no. 36, pp. 13958-13963, 2008.

[26] C. Novo, A. M. Funston, I. Pastoriza-Santos, L. M. Liz-Marzán, and P. Mulvaney, "Influence of the medium refractive index on the optical properties of single gold triangular prisms on a substrate," Journal of Physical Chemistry C, vol. 112, no. 1, pp. 37, 2008.

[27] E. Martinsson, M. M. Shahjamali, K. Enander et al., "Local refractive index sensing based on edge gold-coated silver nanoprisms," Journal of Physical Chemistry C, vol. 117, no. 44, pp. 23148-23154, 2013.

[28] S. Samanta, P. Sarkar, S. Pyne, G. P. Sahoo, and A. Misra, "Synthesis of silver nanodiscs and triangular nanoplates in PVP matrix: Photophysical study and simulation of UV-vis extinction spectra using DDA method," Journal of Molecular Liquids, vol. 165, pp. 21-26, 2012.

[29] P. Sarkar, D. K. Bhui, H. Bar et al., "DDA-based simulation of UV-vis extinction spectra of Ag nanorods synthesized through seed-mediated growth process," Plasmonics, vol. 6, no. 1, pp. 4351, 2011.

[30] L. P. Jiang, S. Xu, J. Zhu, J. Zhang, and H. Chen, "Ultrasonicassisted synthesis of monodisperse single-crystalline silver nanoplates and gold nanorings," Inorganic Chemistry, vol. 43, no. 19, pp. 5877-5883, 2004.

[31] B. Tang, J. An, X. Zheng et al., "Silver nanodisks with tunable size by heat aging," The Journal of Physical Chemistry C, vol. 112, no. 47, pp. 18361-18367, 2008.

[32] K. L. Kelly, E. Coronado, L. L. Zhao, and G. C. Schatz, "The optical properties of metal nanoparticles: the influence of size, shape, and dielectric environment," The Journal of Physical Chemistry B, vol. 107, no. 3, pp. 668-677, 2003.

[33] S. L. Zhu, C. L. Du, and Y. Q. Fu, "Localized surface plasmon resonance-based hybrid Au-Ag nanoparticles for detection of Staphylococcus aureus enterotoxin B," Optical Materials, vol. 31, no. 11, pp. 1608-1613, 2009.

[34] L. J. Sherry, S. Chang, G. C. Schatz, R. P. Van Duyne, B. J. Wiley, and Y. Xia, "Localized surface plasmon resonance spectroscopy of single silver nanocubes," Nano Letters, vol. 5, no. 10, pp. 20342038, 2005 .

[35] P. C. Chaumet, A. Rahmani, and G. W. Bryant, "Generalization of the coupled dipole method to periodic structures," Physical Review B, vol. 67, Article ID 165404, 2009. 

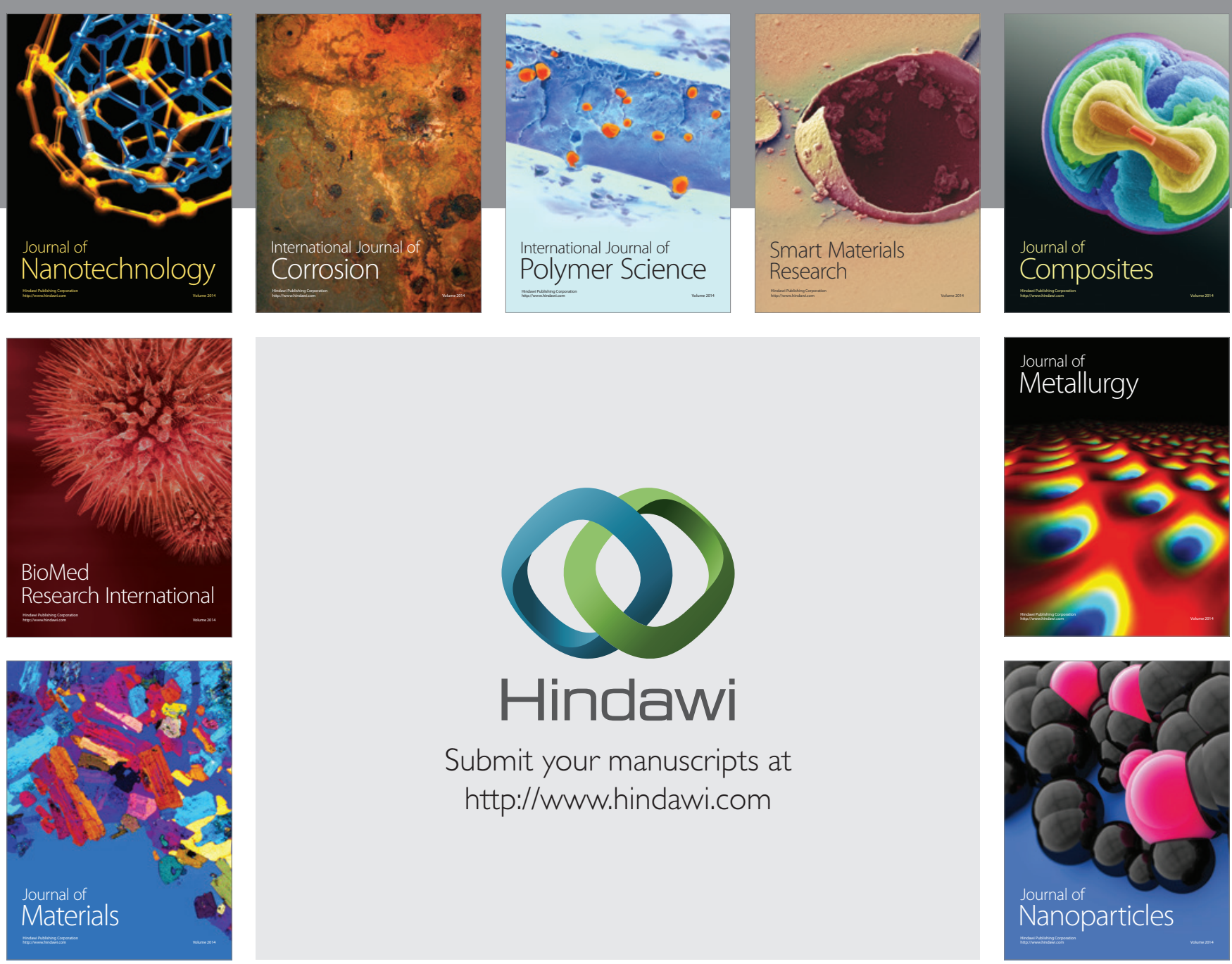

Submit your manuscripts at http://www.hindawi.com
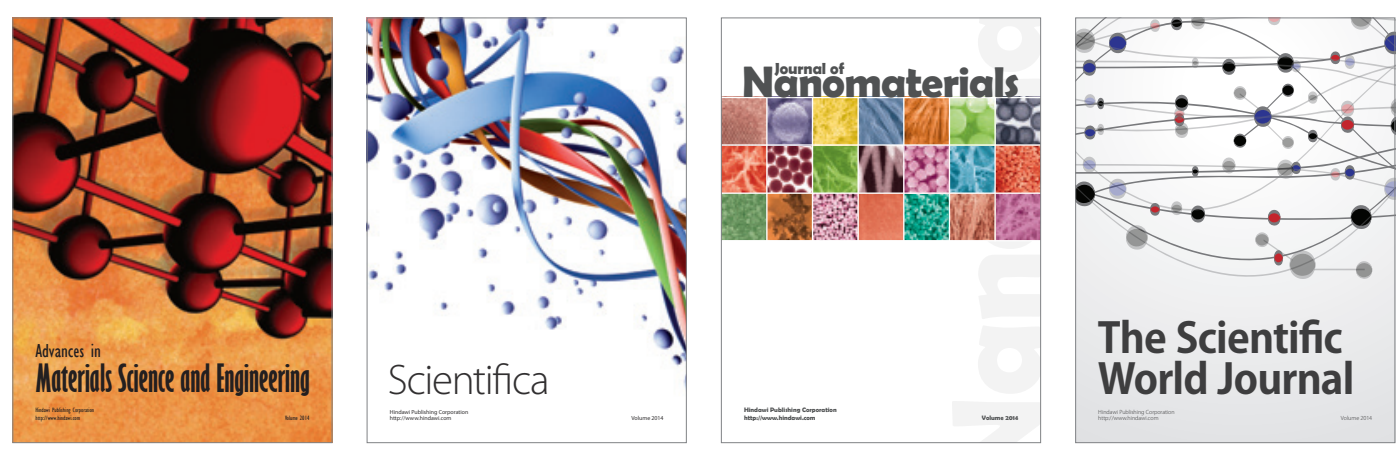

\section{The Scientific World Journal}
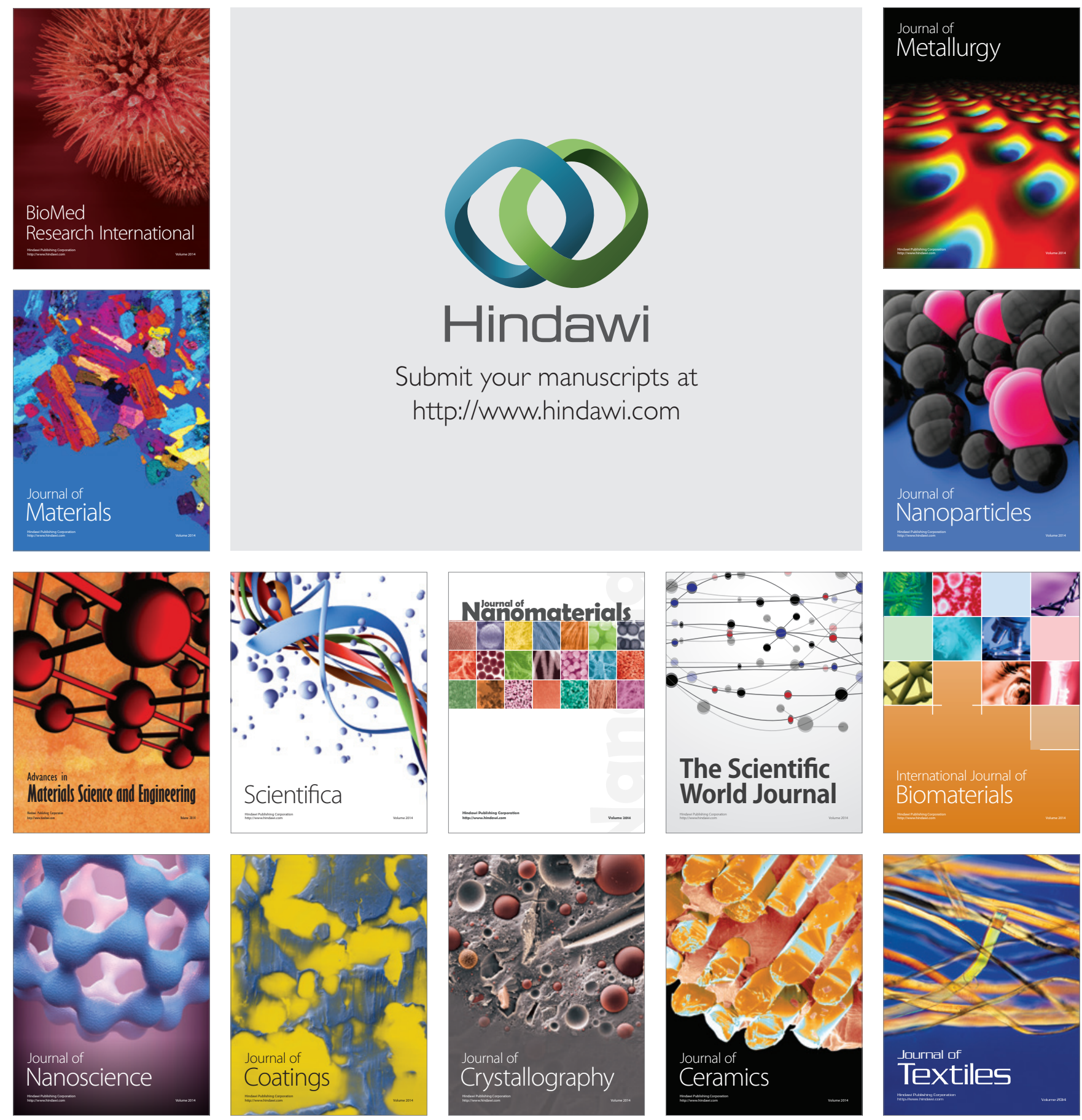